Artigo Original

Original Article

Rafaella Cristina Oliveira

Letícia Caldas Teixeira ${ }^{1}$

Ana Cristina Côrtes Gama ${ }^{1}$

Adriane Mesquita de Medeiros ${ }^{2}$

Descritores

Voz

Distúrbios da voz

Disfonia/epidemiologia

Percepção auditiva

Criança

Keywords

Voice

Voice disorders

Dysphonia/epidemiology

Auditory perception

Child

Endereço para correspondência:

Rafaella Cristina Oliveira

R. Benjamim Brandão, 60, Paraíso, Belo Horizonte (MG), Brasil, CEP: 30270-160.

E-mail: rafaellacris_bh@hotmail.com

Recebido em: 12/6/2010

Aceito em: 5/4/2011

\section{Análise perceptivo-auditiva, acústica e autopercepção vocal em crianças}

\author{
Auditory-perceptive, acoustic and vocal self-perception \\ analyses in children
}

\section{RESUMO}

Objetivo: Estabelecer a ocorrência de crianças com disfonia e relacionar os dados das análises perceptivoauditiva, acústica e de autopercepção vocal de crianças com e sem disfonia. Métodos: Participaram 70 crianças, na faixa etária entre 6 e 10 anos de idade, sendo 37 do gênero feminino e 33 do gênero masculino. Foi gravada a emissão sustentada da vogal /a/ e as crianças responderam à pergunta "O que você acha da sua voz?". Posteriormente, foi realizada a análise perceptivo-auditiva das vozes, com base nos parâmetros da escala GRBASI. Além disso, foi realizada a análise acústica, considerando as medidas de frequência fundamental, quociente de perturbação de frequência e amplitude e proporção harmônico-ruído. A análise da autopercepção vocal das crianças foi fundamentada em análise de conteúdo. Os dados foram submetidos à análise estatística pertinente. Resultados: A ocorrência de crianças com disfonia foi de 37,14\%. A soprosidade foi a qualidade vocal mais comum entre as crianças disfônicas, seguida de rugosidade, que também foi frequente. As medidas acústicas de quociente de perturbação de frequência e amplitude e a proporção harmônico ruído foram mais elevadas entre as crianças disfônicas. Entretanto, tais medidas foram semelhantes entre as crianças que apresentaram percepção positiva e negativa em relação à voz. A autopercepção vocal negativa foi maior entre as crianças com disfonia. Conclusão: A ocorrência de disfonia no grupo de crianças estudado é de 37,14\%. Crianças disfônicas apresentam autopercepção vocal negativa, voz predominantemente soprosa e/ou rugosa, além de medidas acústicas alteradas, quando comparadas a crianças não disfônicas.

\begin{abstract}
Purpose: To establish the occurrence of dysphonic children and to relate data from auditory-perceptive, acoustic and vocal self-perception analyses of dysphonic and non-dysphonic children. Methods: Participants were 70 children, 37 female and 33 male, with ages ranging from 6 to 10 years. The sustained emission of the vowel /a/ was recorded, and children replied to the question "What do you think of your voice?". After that, the auditory-perceptive analysis of their voices was carried out, based on the parameters of the GRBASI scale. The acoustic analysis was also conducted, considering the following measures: fundamental frequency, frequency and amplitude perturbation quotient, and harmonic-to-noise ratio. The self-perception analysis of the subjects' voices was based on content analysis. Data were statistically analyzed. Results: The occurrence of dysphonic children was $37.14 \%$. Breathiness was the most common vocal quality among dysphonic children, followed by roughness, which was also common. The acoustic measures frequency and amplitude perturbation quotient and harmonic-to-noise ration were higher among dysphonic children. However, these measures were similar between children that had positive self-perception and those with negative self-perception. Negative self-perception was more frequent among dysphonic children. Conclusion: The occurrence of dysphonia in the studied group was $37.14 \%$. Dysphonic children present negative self-perception of their voices, voice quality predominantly rough and/or breathy, and altered acoustic measures, when compared to non-dysphonic children.
\end{abstract}

Trabalho realizado na Universidade Federal de Minas Gerais - UFMG - Belo Horizonte (MG), Brasil.

(1) Curso de Fonoaudiologia, Universidade Federal de Minas Gerais - UFMG - Belo Horizonte (MG), Brasil.

(2) Curso de Educação Física, Centro Universitário de Belo Horizonte - UNI-BH - Belo Horizonte (MG), Brasil. 


\section{INTRODUÇÃO}

No decorrer da vida, mudanças vocais vão acontecer e acompanhar o desenvolvimento do indivíduo, do ponto de vista físico, psicológico e social. O desenvolvimento da laringe ocorre de maneira intensa e contínua desde o nascimento do bebê $\hat{e}^{(1,2)}$.

Até a idade entre 6 e 7 anos, as vozes de meninos e meninas são muito próximas quanto à frequência fundamental. As diferenças na qualidade vocal podem ser atribuídas a modelos vocais divergentes, com base nos quais as meninas buscam uma voz feminina típica com qualidade vocal mais soprosa e os meninos, uma qualidade vocal mais hiperfuncional ${ }^{(3)}$.

$\mathrm{Na}$ infância, os quadros de disfonia ocorrem em decorrência de desajustes na fonação, que podem estar relacionados ao comportamento vocal inadequado ou a um desequilíbrio dos fatores anatômicos, fisiológicos, sociais, emocionais e/ou ambientais. Muitas vezes, a disfonia apresenta-se associada a outras etiologias, que variam desde afecções autolimitantes, como as laringites virais agudas, até lesões incapacitantes e com risco de vida, como tumores ou estenoses laríngeas de graus variados ${ }^{(4,5)}$.

Estudos epidemiológicos recentes mostram que a disfonia na infância acomete de $6 \%$ a $38 \%$ de crianças, sendo a rouquidão e a soprosidade as alterações vocais mais frequentes ${ }^{(6-9)}$.

Historicamente a disfonia infantil é descrita com maior frequência em meninos devido as diferenças de personalidade e à prática de atividades físicas e sociais, que demandam uso vocal excessivo $^{(10,11)}$. Entretanto, atualmente é crescente o aumento do número de casos de disfonia entre meninas, em faixas etárias cada vez menores. De acordo com a literatura, a socialização cada vez mais precoce, a maior exposição a ambientes ruidosos e a participação de ambos os gêneros em atividades esportivas e de canto são fatores que provavelmente têm contribuído para essas mudanças ${ }^{(12)}$.

A disfonia infantil deve ser investigada precocemente, pois quanto antes for estabelecido o diagnóstico e o início do tratamento, menor será o impacto na comunicação.

Para viabilizar a avaliação e o atendimento dos casos de disfonia, a clínica fonoaudiológica dispõe de uma série de possibilidades de análise da produção e da percepção do sinal vocal. Algumas análises baseiam-se no método de percepção auditiva do terapeuta treinado e contam com escalas padronizadas de avaliação, tais como GRBASI ${ }^{(13)}$. Trata-se de método subjetivo de análise vocal, por depender do julgamento do(s) avaliador(es). Por isso, gera discussões entre os profissionais que atuam na área da voz. No entanto, é indiscutível a sua contribuição na prática clínica ${ }^{(14)}$.

A análise acústica, outro método de avaliação vocal, é objetiva, não invasiva e propicia a integração dos dados da avaliação perceptivo-auditiva com o plano fisiológico. Ela detalha o processo de geração do sinal sonoro e fornece uma estimativa indireta dos padrões vibratórios das pregas vocais, bem como dos formatos do trato vocal e de suas modificações ${ }^{(15)}$.

Além dessas avaliações, os protocolos que investigam a autopercepção do indivíduo sobre a qualidade de vida e da voz, quando associados às várias outras formas de avaliações, contribuem para o raciocínio sobre o diagnóstico do paciente nos quadros de disfonia ${ }^{(13)}$. Vale ressaltar que nos casos de disfonia infantil a problemática do diagnóstico é maior, já que crianças sem problema de voz podem apresentar grau discreto de nasalidade, rouquidão e soprosidade ${ }^{(1)}$.

Considerando que as alterações vocais na infância podem comprometer o desenvolvimento de uma comunicação efetiva no âmbito social ${ }^{(5)}$, faz-se pertinente a realização de pesquisas delineando peculiaridades da disfonia infantil. Acreditamos que este estudo alicerça ainda mais o trabalho clínico fonoaudiológico, na perspectiva que ressalta a importância da correlação entre as análises perceptivo-auditiva, acústica e de autopercepção vocal das crianças para a compreensão da voz infantil.

O objetivo deste estudo foi estabelecer a ocorrência de crianças com disfonia e relacionar os dados obtidos nas análises perceptivo-auditiva, acústica e de autopercepção vocal de crianças disfônicas e não disfônicas.

\section{MÉTODOS}

Esta pesquisa consiste em um estudo transversal com amostra aleatória, aprovado na Comissão de Ética em Pesquisa da Universidade Federal de Minas Gerais (UFMG), sob número 676/08. A amostra foi composta por 70 crianças, na faixa etária entre 6 e 10 anos de idade, sendo 37 do gênero feminino (média de idade de 8,35 anos, $\mathrm{DP}=1,29)$, e 33 do gênero masculino (média de idade de 7,7 anos, $\mathrm{DP}=1,44$ ). As crianças eram estudantes de primeira a quinta série do ensino fundamental do Centro Pedagógico da UFMG, em Belo Horizonte (MG), Brasil.

O Centro Pedagógico é uma escola da rede pública e está implantada no campus da Universidade. A seleção das crianças, desde a década de 1990, é feita por meio de sorteio. Os pais inscrevem seus filhos e acompanham o processo. Assim, a escola abrange crianças de todas as classes sociais.

Todas as crianças foram autorizadas pelos responsáveis, por meio da assinatura do Termo de Consentimento Livre e Esclarecido (TCLE). Os critérios de exclusão foram: crianças com faixa etária inferior a 6 anos ou superior a 10 anos e 11 meses (excluindo-se, dessa forma, o período da muda vocal), não estudantes do Centro Pedagógico, portadoras de distúrbio neurológico ou perda auditiva diagnosticada ou que não compreendessem as solicitações ou a pergunta feita pela pesquisadora.

A pesquisa foi desenvolvida em quatro etapas:

Primeira etapa - Gravação das vozes das crianças. Os registros vocais foram coletados individualmente, utilizando um gravador digital profissional (DAT - Digital Audio Tape) da marca Sony ${ }^{\circledR}$, equipado com microfone unidirecional. O microfone foi posicionado a $10 \mathrm{~cm}$ de distância da boca do falante e com ângulo de captação direcional de $90^{\circ}$, para evitar captação de ruído ambiental. As sessões de gravação tiveram duração aproximada de 10 minutos e ocorreram em horários previamente agendados com a coordenadora da escola. Para a gravação, foi utilizada uma sala silenciosa, com nível de ruído inferior a 50 dBNPS (nível de pressão sonora), aferido por meio de um medidor de nível de pressão sonora digital. A criança foi orientada a realizar duas tarefas básicas para a gravação da voz: fazer a emissão sustentada 
da vogal /a/ em tempo máximo de fonação; e responder à pergunta "O que você acha da sua voz?". A compreensão da criança foi garantida pela observação da resposta, obedecendo ao critério de exclusão do estudo.

- Segunda etapa - Análise perceptivo-auditiva das vozes das crianças com base nos parâmetros estabelecidos pela escala GRBASI. As juízas avaliaram o grau geral da alteração (G), que foi dividido em: G0 (ausência de disfonia); G1 (disfonia leve); G2 (disfonia moderada) e G3 (disfonia intensa) e a predominância da qualidade vocal, dividida em: rugosidade $(\mathrm{R})$, soprosidade (B), astenia (A), tensão (T) e instabilidade vocal (I).

Para esta avaliação, colaboraram três fonoaudiólogas especialistas em voz. As fonoaudiólogas ouviram a gravação da emissão da vogal /a/ sustentada, definida por fornecer informações da fonte sonora ${ }^{(16)}$. As análises foram feitas separadamente. As avaliadoras utilizaram fones de ouvido e ouviram cada uma das amostras por três vezes, em local silencioso. A partir disso, preencheram a folha de registro da avaliação perceptivo-auditiva referente a cada uma das crianças. Todas as avaliadoras que participaram do estudo tinham treinamento prévio em análise perceptivo-auditiva de vozes infantis e com a utilização da escala GRBASI.

Para análise da concordância intra-avaliador, foram repetidas 14 amostras (20\%), escolhidas aleatoriamente entre aquelas que foram apresentadas em ordem randomizada. As três avaliadoras apresentaram confiabilidade superior a $75 \%$, calculada por meio do teste estatístico Kappa. As vozes das crianças foram consideradas alteradas ( $G$ maior ou igual a 1) quando houve concordância entre pelo menos duas avaliadoras. Os resultados das análises dos demais parâmetros da qualidade vocal foram determinados pelo resultado mais prevalente entre as avaliações. Em nenhum momento se observou uma avaliação discordante entre as três avaliadoras, ou seja, pelo menos dois avaliadores analisaram da mesma forma um parâmetro perceptivo-auditivo de uma mesma voz. A concordância de pelo menos duas avaliadoras foi o critério utilizado para aumentar a confiabilidade da análise perceptivo-auditiva e, consequentemente, da seleção da amostra.

- Terceira etapa - Análise acústica das vozes das crianças utilizando a emissão sustentada da vogal /a/. Foram avaliados os seguintes parâmetros: frequência fundamental $\left(\mathrm{F}_{0}\right)$, quociente de perturbação de amplitude (APQ\%), quociente de perturbação de frequência (PPQ\%), proporção harmônico-ruído (PHR dB). A gravação da vogal /a/ sustentada foi transferida para o PC Dell® Pentium (R), placa de som Sound Blaster Live 24-bit, pré-amplificador Behringer Mic200, e a onda sonora foi gravada no módulo MDVP (Multi-Dimensional Voice Program Advanced) do programa Multi-speech model 3700 da Kay Elemetrics®. O início e o final da emissão foram eliminados, devido a suas características irregulares. Não há valores de referência como data base para a análise acústica das vozes infantis no programa MDVP. Dessa forma, a análise comparativa foi realizada entre as crianças disfônicas e não disfônicas. Para a caracterização das crianças disfônicas e não disfônicas foram considerados os valores de $\mathrm{G}$ da análise preceptivo- auditiva, sendo que os valores de $\mathrm{G}$ iguais ou superiores a 1 indicaram a presença de disfonia.

- Quarta etapa - Análise qualitativa da autopercepção das crianças acerca de suas vozes utilizando a amostra de fala encadeada. A análise dos dados foi fundamentada na análise de conteúdo, proposta por $\operatorname{Bardin}^{(17)}$, em que as ideias centrais das respostas de uma mesma questão foram identificadas para posterior comparação do grupo e sintetização do conhecimento geral. Após a seleção das categorias a serem analisadas, verificou-se a sua frequência de ocorrência (aparecimento) nos discursos dos sujeitos. Para esta avaliação, a autopercepção vocal das crianças foi considerada positiva ou negativa. A autopercepção foi considerada positiva quando a criança relatou que a voz é "bonita, legal, boa, transmite alegria e gosta da voz". A autopercepção negativa foi considerada quando a criança relatou que a voz é "ruim, feia, transmite braveza e não gosta da voz".

Os dados foram armazenados e tabulados. Para a análise estatística, foi utilizado software específico, o SPSS, versão 15. Inicialmente, foram feitas análises descritivas dos dados, com cálculo de médias, desvios-padrões (DP), mediana, mínimo e máximo, para as variáveis contínuas. Para as variáveis categóricas, esta análise consistiu em determinar a frequência e respectivo percentual de cada classe.

Foi realizado os testes de hipóteses $t$ de Student, para comparar as médias dos parâmetros da análise acústica nas crianças disfônicas e não disfônicas, e testes de associação entre a presença ou ausência de disfonia e as variáveis gênero e autopercepção vocal. Adotamos o nível de significância de $5 \%$. No caso específico do teste de associação, utilizou-se o teste Qui-quadrado quando todos os cruzamentos tinham cinco ou mais observações ou o Teste Exato de Fisher quando todos os cruzamentos tinham menos de cinco observações.

Após a análise dos dados, realizou-se devolutiva para a escola e responsáveis acerca dos resultados obtidos. As crianças com disfonia receberam orientações sobre saúde vocal, assim como seus responsáveis. Foi realizado encaminhamento à Unidade Básica de Saúde para os interessados.

\section{RESULTADOS}

A ocorrência de crianças com disfonia encontrada nesta pesquisa foi de $37,14 \%$ ( $n=26$ ), considerando a análise da vogal /a/ sustentada. Além disso, o número de casos de disfonia foi semelhante entre os gêneros feminino e masculino (Tabela 1).

Tabela 1. Ocorrência de disfonia em crianças, de acordo com o gênero

\begin{tabular}{|c|c|c|c|c|c|c|}
\hline \multirow{3}{*}{ Vogal } & \multicolumn{4}{|c|}{ Gênero } & \multirow{3}{*}{ Total } & \multirow{3}{*}{$\begin{array}{l}\text { Valor } \\
\text { de } p\end{array}$} \\
\hline & \multicolumn{2}{|c|}{ Feminino } & \multicolumn{2}{|c|}{ Masculino } & & \\
\hline & $\mathrm{n}$ & $\%$ & $\mathrm{n}$ & $\%$ & & \\
\hline Não disfônicas & 23 & 32,86 & 21 & 30,00 & 44 & 1,0 \\
\hline Disfônicas & 14 & 20,00 & 12 & 17,14 & 26 & \\
\hline Total & 37 & 52,86 & 33 & 47,14 & 70 & \\
\hline
\end{tabular}

Teste Qui-quadrado $(p \leq 0,05)$ 
Dentre as 26 crianças disfônicas, 84,60\% $(\mathrm{n}=22)$ apresentaram alteração de grau leve $(\mathrm{G} 1), 7,80 \%(\mathrm{n}=2)$ de grau moderado (G2) e 7,80\% (n=2) de grau intenso (G3).

Em relação às características perceptivo-auditivas, a soprosidade (B) foi a qualidade vocal mais comum $(n=25)$ entre as 26 crianças disfônicas, seguida de rugosidade $(\mathrm{R})$, que também foi frequente $(\mathrm{n}=18)$. Os demais parâmetros perceptivo-auditivos da escala GRBASI não foram observados pelos avaliadores (Tabela 2).

Tabela 2. Resultados da escala GRBASI para as crianças disfônicas

\begin{tabular}{lcc}
\hline & $\mathrm{n}$ & $\%$ \\
\hline (G) Grau $\geq 1$ & 26 & 100,00 \\
(R) Rugosidade & 18 & 69,23 \\
(B) Soprosidade & 25 & 96,15 \\
(A) Astenia & 0 & 00,00 \\
(S) Tensão & 0 & 00,00 \\
(I) Instabilidade & 0 & 00,00 \\
\hline
\end{tabular}

Quanto às medidas acústicas, considerando a amostra total, as médias obtidas foram: $\mathrm{F}_{0}=240,17 \mathrm{~Hz}, \mathrm{PPQ} \%=1,12 \%$, $\mathrm{APQ} \%=2,26 \%$ e PHR $\mathrm{dB}=0,13 \mathrm{~dB}$. Houve diferença na comparação de crianças disfônicas e não disfônicas quanto a: quociente de perturbação de frequência (PPQ\%), quociente de perturbação de amplitude (APQ\%) e proporção harmônicoruído (PHR dB) (Tabela 3).

A média do PPQ\% foi maior nas crianças disfônicas. O valor médio obtido para crianças não disfônicas foi de $0,82 \%$ e o para crianças disfônicas foi de 1,34\%. A média do APQ\% foi menor nas crianças não disfônicas. Em relação aos valores da média de PHR $\mathrm{dB}$, estes foram maiores entre as crianças disfônicas (Tabela 3).

A maior parte das crianças demonstrou autopercepção vocal positiva. A autopercepção negativa foi maior entre as crianças disfônicas, havendo diferença em relação às não disfônicas. Dentre as 26 crianças disfônicas, 9 (34,61\%) apresentaram percepção negativa acerca da própria voz (Tabela 4).

As médias das medidas acústicas ( $\mathrm{F}_{0}, \mathrm{APQ}, \mathrm{PPQ}$ e PHR) foram semelhantes entre as crianças que apresentaram autopercepção positiva e negativa em relação à voz, não havendo diferença entre os grupos (Tabela 5).

\section{DISCUSSÃO}

A disfonia infantil causa impacto negativo na saúde geral da criança, bem como no desenvolvimento de uma comunicação adequada. De modo específico, as crianças disfônicas são julgadas de maneira mais negativa por seus pares do que as crianças não disfônicas, em relação às características bipolares, físicas e de personalidade, como sujo/limpo, mal/bom, doente/ saudável, triste/feliz e errado/correto ${ }^{(5)}$.

Estudos epidemiológicos revelam que a prevalência de alteração vocal na infância varia de $6 \%$ a $38 \%{ }^{(6-8)}$. Tais dados são corroborados pelos índices obtidos nesta pesquisa. No

Tabela 3. Análise acústica para crianças disfônicas e não disfônicas

\begin{tabular}{|c|c|c|c|c|c|c|c|c|c|c|c|c|c|}
\hline \multirow[t]{2}{*}{ Variáveis } & \multicolumn{2}{|c|}{ Média } & \multicolumn{2}{|c|}{ DP } & \multicolumn{2}{|c|}{ Mediana } & \multicolumn{2}{|c|}{ Mínimo } & \multicolumn{2}{|c|}{ Máximo } & \multicolumn{2}{|c|}{ CV Pearson } & \multirow[t]{2}{*}{ Valor de $p$} \\
\hline & $\mathrm{D}$ & ND & $\mathrm{D}$ & ND & $\mathrm{D}$ & ND & $\mathrm{D}$ & ND & D & ND & $\mathrm{D} \%$ & $\mathrm{ND} \%$ & \\
\hline $\mathrm{F}_{0}(\mathrm{~Hz})$ & 230,30 & 244,69 & 39,63 & 28,04 & 228,67 & 243,92 & 121,70 & 187,07 & 323,07 & 312,08 & 17,21 & 11,46 & 0,321 \\
\hline PPQ\% & 1,34 & 0,82 & 0,68 & 0,6 & 1,20 & 0,63 & 0,49 & 0,13 & 2,96 & 2,24 & 50,61 & 73,72 & $0,001^{*}$ \\
\hline APQ\% & 2,70 & 1,72 & 1,20 & 0,48 & 2,37 & 1,64 & 1,05 & 1,00 & 5,59 & 2,76 & 44,47 & 27,91 & $0,001^{*}$ \\
\hline PHR (dB) & 0,14 & 0,11 & 0,03 & 0,03 & 0,14 & 0,11 & 0,06 & 0,04 & 0,22 & 0,15 & 24,29 & 24,84 & $0,001^{*}$ \\
\hline
\end{tabular}

* Valores significativos $(p \leq 0,05)-$ Teste t de Student

Legenda: $F_{0}=$ frequência fundamental; $A P Q \%$ = quociente de perturbação de amplitude a curto prazo; $P P Q \%=$ quociente de perturbação de frequência a curto prazo; $\mathrm{PHR}=$ proporção harmônico-ruído; $\mathrm{D}$ = indivíduo disfônico; ND = indivíduo não disfônico; $\mathrm{DP}=$ desvio-padrão; CV Pearson = coeficiente de variabilidade de Pearson

Tabela 4. Autopercepção vocal de crianças disfônicas e não disfônicas

\begin{tabular}{|c|c|c|c|c|c|}
\hline \multirow[t]{2}{*}{ Fala } & \multicolumn{2}{|c|}{ Disfônicas } & \multicolumn{2}{|c|}{ Não disfônicas } & \multirow[t]{2}{*}{ Valor de $p$} \\
\hline & $\mathrm{n}$ & $\%$ & $\mathrm{n}$ & $\%$ & \\
\hline Negativa & 9 & 34,61 & 2 & 4,55 & $0,001^{*}$ \\
\hline Positiva & 17 & 65,38 & 42 & 95,54 & \\
\hline
\end{tabular}

*Valores significativos $(\mathrm{p} \leq 0,05)-$ Teste Exato de Fisher

Tabela 5. Relação entre dados de análise acústica e autopercepção vocal das crianças

\begin{tabular}{|c|c|c|c|c|c|c|c|}
\hline \multirow[t]{2}{*}{ Variáveis } & \multicolumn{3}{|c|}{ Autopercepção positiva } & \multicolumn{3}{|c|}{ Autopercepção negativa } & \multirow[t]{2}{*}{ Valor de $p$} \\
\hline & $\mathrm{n}$ & Média & DP & $\mathrm{n}$ & Média & DP & \\
\hline $\mathrm{F}_{0}(\mathrm{~Hz})$ & 59 & 241,79 & 29,66 & 11 & 231,49 & 43,26 & 0,331 \\
\hline PPQ\% & 59 & 1,09 & 0,57 & 11 & 1,30 & 0,89 & 0,317 \\
\hline APQ\% & 59 & 2,15 & 0,86 & 11 & 2,81 & 1,33 & 0,138 \\
\hline PHR (dB) & 59 & 0,12 & 0,03 & 11 & 0,14 & 0,04 & 0,098 \\
\hline
\end{tabular}

Teste t para igualdade de médias $(p \leq 0,05)$

Legenda: $F_{0}=$ frequência fundamental; $A P Q \%$ = quociente de perturbação de amplitude a curto prazo; $P P Q \%$ = quociente de perturbação de frequência a curto prazo; $\mathrm{PHR}=$ proporção harmônico-ruído; $\mathrm{DP}$ = desvio-padrão 
entanto, os resultados do presente estudo divergem dos que foram obtidos em pesquisa realizada com crianças de 6 a 10 anos de idade, em que a prevalência de alteração vocal variou de $59 \%$ a $64 \%{ }^{(9)}$.

Ao relacionar a variável gênero com a presença ou não de disfonia, pesquisadores enfatizam que a disfonia infantil ocorre com maior frequência em meninos. Isso ocorre porque eles possuem fator de risco para o desenvolvimento da disfonia, devido a diferenças de personalidade e à prática de atividades físicas e sociais, que demandam uso vocal excessivo ${ }^{(10,11,18)}$. Entretanto, tanto neste estudo quanto em outra pesquisa semelhante ${ }^{(8)}$ essa diferença não foi observada.

É importante destacar que as configurações laríngeas de meninas e meninos são semelhantes ${ }^{(3)}$. Além disso, atualmente as meninas têm demonstrado maior interesse por brincadeiras agitadas e agressivas, que antes eram mais observadas entre os meninos. Essas atividades requerem competição sonora além de utilização indevida e abusiva da voz, atitudes que podem gerar hiperfuncionamento e distúrbios laríngeos ${ }^{(13,19)}$.

Nas escolas, em geral, as crianças têm muitas oportunidades para cometer abusos vocais, pois participam de atividades ao ar livre, em que o autocontrole vocal é dificultado. Outra consideração é que crianças apresentam com frequência quadros de respiração oral, alergias respiratórias e infecções nas vias aéreas superiores. Esses fatores, quando articulados, fazem com que a utilização da voz se dê em condições inadequadas, na presença de mucosas ressecadas, edema e irritação do trato vocal $^{(5)}$.A escala GRBASI é um dos instrumentos de avaliação vocal $^{(13)}$. Autores ${ }^{(20)}$ observaram que este instrumento é válido para a avaliação das vozes infantis, embora se deva considerar a importância de avaliadores treinados, devido ao alto nível de subjetividade envolvido no processo.

Neste estudo, a seleção de três juízes com índice de concordância intra-avaliador superior a 75\% para análise perceptivoauditiva da escala GRBASI diminuiu a subjetividade do processo. É importante ressaltar que o teste-reteste de segurança da escala GRBASI é moderado ${ }^{(13)}$.

No que se refere às características perceptivo-auditivas, a soprosidade (B) foi a qualidade vocal mais observada nesta pesquisa. Outros autores também concluíram que esta é a qualidade vocal mais comum em crianças ${ }^{(3,9)}$. Estudos futuros são necessários para o melhor entendimento de quais fatores anatômicos e/ou funcionais podem estar relacionados com a presença de soprosidade na voz de crianças disfônicas.

A rugosidade $(\mathrm{R})$ também foi uma alteração vocal frequente entre as crianças disfônicas. Este dado é esperado, uma vez que ela é favorecida pelo mau uso ou abuso vocal, principalmente quando se refere à laringe da criança ${ }^{(1)}$. Além disso, muitas doenças laríngeas infantis têm a rouquidão como seu principal indicador ${ }^{(1)}$.

Em relação à análise acústica, após realizar estudo de revisão, pesquisadores constataram que muitos trabalhos reconhecem pouca diferenciação entre vozes normais e disfônicas ${ }^{(20)}$. Há necessidade um maior número de pesquisas sobre o assunto, com maior padronização e normatização de medidas ${ }^{(13,21)}$.

É importante destacar que os valores médios obtidos nas medidas acústicas $\left(\mathrm{F}_{0}, \mathrm{APQ}, \mathrm{PPQ}\right.$ e PHR) discordaram dos valores referidos em outros estudos ${ }^{(21-23)}$. Provavelmente, isso esteja relacionado às diferenças nos procedimentos de registro e cálculos matemáticos utilizados para as análises. As medidas acústicas variam de acordo com o programa utilizado, limitando a comparação direta entre estudos que utilizaram diferentes $\operatorname{programas}^{(1,17)}$.

A média de $\mathrm{F}_{0}(240,17 \mathrm{~Hz})$ obtida nesta pesquisa foi semelhante às obtidas na maior parte dos estudos ${ }^{(24,25)}$. A médias de $230,30 \mathrm{~Hz}$, obtida em crianças disfônicas, e $244,69 \mathrm{~Hz}$, obtida em não disfônicas, também corroboram dados da literatura ${ }^{(22)}$.

A medida de jitter é o quociente de perturbação de frequência (PPQ\%) que se relaciona com a perturbação da frequência de vibração das pregas vocais em curto prazo ${ }^{(1,22)}$. A medida de shimmer é o quociente de perturbação de amplitude (APQ\%) calculado a partir da média das perturbações de amplitude, ciclo a ciclo, em toda a amostra vocal ${ }^{(1,11)}$.

As grandes variações das medidas de jitter e shimmer nas crianças são sugestivas de mudanças anatomofisiológicas das estruturas laríngeas e falta de controle laríngeo ${ }^{(20)}$. Destaca-se que a prega vocal infantil ainda não sofreu o processo de diferenciação das camadas intermediária e profunda da lâmina própria $^{(3)}$. Neste estudo, o valor das médias encontradas para as medidas de $\mathrm{PPQ} \%$ e APQ\% foram maiores para crianças disfônicas. Estes achados corroboram os resultados da maior parte dos estudos na área ${ }^{(23,26)}$.

No que se refere ao PPQ\%, autores verificaram elevação significativa dos valores de perturbação de frequência em vozes de crianças com nódulos vocais ${ }^{(23)}$. Isso mostra que alterações na estrutura da mucosa afetam a regularidade de vibração ciclo a ciclo da prega vocal, o que resulta no aumento dos valores de jitter ${ }^{(1)}$. Ao contrário, outro estudo obteve valores de jitter que não revelaram diferenças entre vozes normais e disfônicas ${ }^{(21)}$.

Para a medida acústica APQ\%, um estudo verificou aumento nos valores de shimmer em grupo de sujeitos com papilomatose e nódulos vocais ${ }^{(26)}$. Em outras pesquisas, o valor de shimmer não mostrou diferença entre os grupos de crianças com vozes normais e disfônicas ${ }^{(21,22)}$. Destaca-se que a literatura não é unânime quanto ao poder de diferenciação do shimmer.

A proporção harmônico-ruído (PHR dB) é um dos melhores parâmetros de aplicação clínica, tanto na quantificação dos desvios vocais quanto na avaliação dos procedimentos de tratamento da disfonia, apresentando relação direta com a qualidade vocal ${ }^{(1)}$. É considerada uma das medidas de perturbação de ruído que quantificam a porção de ruído em relação à porção de harmônicos em uma amostra vocal, podendo ser de grande valor na diferenciação entre vozes normais e disfônicas ${ }^{(1,20)}$.

Em relação às medias $\mathrm{PHR} \mathrm{dB}$, observou-se valores maiores entre as crianças disfônicas. Tais resultados discordam de estudo $^{(23)}$ que não evidenciou aumento significativo de PHR dB quando foram comparados grupos de crianças com vozes normais e alteradas.

Apesar de os resultados da análise perceptivo-auditiva e acústica não serem diretamente proporcionais ${ }^{(15)}$, neste estudo observou-se que as crianças classificadas como disfônicas apresentaram maiores valores médios de medidas acústicas. Isso mostra que quanto maior a presença de ruído à emissão, maior a periodicidade de frequência e amplitude no sinal acústico. 
Considerando a natureza multidimensional da disfonia, a utilização conjunta de protocolos de avaliação perceptivoauditiva, acústica e de autopercepção do indivíduo auxilia na compreensão dos quadros ${ }^{(27)}$. Em relação à autopercepção vocal, os resultados encontrados nesta pesquisa reforçam a ideia de que a disfonia é um sintoma pouco percebido pelas crianças ${ }^{(28,29)}$. Isso pôde ser observado pois poucas crianças se autoavaliaram de forma negativa. Entretanto, é preciso destacar que esta autopercepção negativa foi maior entre as crianças disfônicas. Tal evidência está a favor de estudos voltados para criação de protocolos de qualidade de vida em voz específicos para a criança, que buscam estudar mais profundamente este impacto.

As medidas acústicas foram semelhantes entre crianças que apresentaram autopercepção vocal positiva e negativa. $\mathrm{Na}$ literatura compilada não foram encontrados dados que comparam a autopercepção vocal das crianças com as medidas objetivas. Ao se considerar a opinião dos pais em relação à voz de seus filhos, a maior parte deles acredita que as crianças não percebem os problemas de voz porque não referem queixas. No entanto, mencionam que os filhos diminuem o uso da voz diante de alguma alteração vocal ${ }^{(28)}$.

A relação entre os dados das diferentes análises contribui para melhor compreensão do quadro de disfonia em crianças e para a definição da conduta clínica a ser adotada. Deve-se destacar que os dados desta pesquisa reforçam os achados da literatura e contribuem com a prática clínica fonoaudiológica junto a crianças com alterações vocais. Sob um olhar mais abrangente e multifatorial das disfonias infantis, incentiva-se que futuras pesquisas investiguem as condições ambientais das escolas, a fim de se avaliar o quanto elas interferem na produção vocal das crianças. Considera-se importante, também, a construção de projetos para conscientização e esclarecimentos aos pais, educadores e às próprias crianças acerca da importância da voz na comunicação infantil.

\section{CONCLUSÃO}

A ocorrência da disfonia no grupo de crianças estudado é de $37,14 \%$. Crianças disfônicas apresentam autopercepção vocal negativa, voz predominantemente soprosa e/ou rugosa, além de medidas acústicas alteradas, quando comparadas a crianças não disfônicas.

\section{REFERÊNCIAS}

1. Behlau M, Azevedo R, Pontes P. Conceito de voz normal e classificação das disfonias. In: Behlau M, organizador. Voz - O Livro do especialista. 2a ed. Vol.1. Rio de Janeiro: Revinter; 2004.

2. Aronson AE. Clinical voice disorders. New York: Thieme; 1990.

3. McAllister AM, Granqvist S, Sjölander P, Sundberg J. Child voice and noise: a pilot study of noise in day cares and the effects on 10 children's voice quality according to perceptual evaluation. J Voice. 2009;23(5):587-93.

4. Maia AA, Gama AC, Michalick-Triginelli MF. Relação entre transtorno do déficit de atenção/hiperatividade, dinâmica familiar, disfonia e nódulo vocal em crianças. Rev Ciênc Méd (Campinas). 2006;15(5):379-89.

5. Freitas MR, Weckx LL, Pontes PA. Disfonia na infância. Rev Bras Otorrinolaringol. 2000;66(3 Pt 1):257-65.
6. Hirschberg J, Dejonckere PH, Hirano M, Mori K, Schultz-Coulon HJ, Vrticka K. Voice disorders in children. Int J Pediatr Otorhinolaryngol. 1995;32 Suppl:S109-25.

7. Duff MC, Proctor A, Yairi E. Prevalence of voice disorders in African American and European American preschoolers. J Voice. 2004;18(3):348-53.

8. Simões M, Rosa AH, Soares JC, Ribeiro LR, Imamura VM, Bitar ML. Alteração vocal em crianças que frequentam creche. Pró-Fono. 2002;14(3):343-50.

9. Martins AF. Caracterização perceptivo-auditiva da fonte glótica de um grupo de crianças sem queixa vocal [tese]. São Paulo: Pontifícia Universidade Católica de São Paulo; 2002.

10. Pinho SM. Tópicos em voz. Rio de Janeiro: Guanabara Koogan; 2001. Terapia vocal; p.1-18.

11. Carding PN, Roulstone S, Northstone K, ALSPAC Study Team. The Prevalence of childhood dysphonia: a cross-sectional study. J Voice. 2006;20(4):623-30

12. Souza AM, Guerra AF, Pedrosa BF, Franco CF, Fortini MS, Godinho RN, et al. Disfonia na infância: escute a voz do seu paciente! Textos científicos Sociedade Mineira de Pediatria [Internet] 2004 [citado 2004 Ago 28]. Disponível em:http://www.smp.org.br/ ERA 12

13. Bele IV. Reliability in perceptual analysis of voice quality. J Voice. 2005;19(4):555-73.

14. Dornelles S, Jotz GP, Guilherme A. Correlação entre avaliação perceptiva auditiva e nasofibroscopia em crianças sem queixa vocal. Rev AMRIGS. 2007;51(2):121-7.

15. Camargo Z. Avaliação objetiva da voz In: de Angelis CE, Fúria CL, Mourão LH, Kowalski LP. A atuação da fonoaudiologia no câncer de cabeça e pescoço. São Paulo: Lovise; 2000. p. 175-194.

16. Zhang Y, Jiang JJ. Acoustic analyses of sustained and running voices from patients with laryngeal pathologies. J Voice. 2008;22(1):1-9.

17. Bardin L. Análise de conteúdo. Lisboa: Edições 70; 2002.

18. Colombo F. Estudio exploratório sobre la prevalencia de sinfonía infantil em niños de 8 a 12 años: establecimientos escolares [tese]. Rosario: Universidad de Rosário, Facultad de Ciências Médicas; 2000.

19. Denunci F, Diaféria G. Avaliação perceptivo-auditiva da voz de crianças disfônicas através da escala GRBAS. In: X Congresso Brasileiro e II Encontro Mineiro de Fonoaudiologia; 2002; Belo Horizonte. Anais.

20. Sader RC, Hanayama EM. Consideração teóricas sobre a abordagem acústica da voz infantil. Rev CEFAC. 2004;6(3):312-8.

21. Steffen N, Moschetti MB, Wolff C, Dossin L, Oussale M, Keil DT. Frequência fundamental de 131 crianças de 06 a 10 anos estudantes de Porto Alegre relacionada com idade, peso, estatura física e superfície corporal. Rev Soc Bras Fonoaudiol. 1997; 1:22-8.

22. Jotz GP, Cervantes O, Settani FA, Angelis EC. Acoustic measures for the detection of hoarseness in children. Intl Arch Otorhinolaryngol. 2006;10(1):14-20.

23. Campisi P, Tewfik TL, Manoukian JJ, Schloss MD, Pelland-Blais E, Sadeghi N. Computer-assisted voice analysis: establishing a pediatric database. Arch Otolaryngol Head Neck Surg. 2002;128(2):156-60.

24. Cappellari VM, Cielo CA. Características vocais acústicas de crianças pré-escolares. Rev Bras Otorrinolaringol. 2008;74(2):265-72.

25. Braga JN, de Oliveira DS, Sampaio TM. Frequência fundamental da voz de crianças. Rev CEFAC. 2009;11(1):119-26.

26. Niedzieslka G. Acoustic analysis in the diagnostic of voice disorders in children. Int J Pediatr Otorhinolaryngol. 2001;57(3):189-93.

27. Dejonckere PH, Bradley P, Clemente P, Cornut G, Crevier-Buchman L, Friedrich G, et al. A basic protocol for functional assessment of voice pathology, especially for investigating the efficacy of (phonosurgical) treatments and evaluating new assessment techniques. Guideline elaborated by the Committee on Phoniatrics of the European Laryngological Society (ELS). Eur Arch Otorhinolaryngol. 2001;258(2):77-82.

28. Teixeira MZ, Trezza EM, Behlau M. Opinião dos pais sobre a voz de seus filhos de 5 a 12 anos. Rev Paul Pediatr. 2003;21(2)67-75.

29. Hersan RC. Avaliação de voz em crianças. Pró-Fono. 1991;3(1):3-9. 